\title{
Defects and supersolidity: effects of annealing and stress on elastic behavior of solid ${ }^{4} \mathrm{He}$
}

\author{
A. Syshchenko, J. Day, and J. Beamish \\ Department of Physics, University of Alberta, Edmonton, Alberta T6G 2G7, Canada \\ E-mail: beamish@phys.ualberta.ca
}

Received December 3, 2007

\begin{abstract}
Recent measurements showed that solid ${ }^{4} \mathrm{He}$ can decouple from a torsional oscillator below $200 \mathrm{mK}$ and defects appear to be crucial to this behavior. Helium's shear modulus increases in the same range, which can be understood in terms of dislocations pinned by ${ }^{3} \mathrm{He}$ impurities at the lowest temperatures, but mobile above $100 \mathrm{mK}$. We have measured helium's pressure and shear modulus to study the effects of annealing and stresses applied at low temperatures. Pressure gradients produced during crystal growth or plastic deformation are greatly reduced by annealing, but only at temperatures close to melting. Annealing does not change the low-temperature modulus but usually raises it at high temperature, as expected if annealing eliminates some dislocations. Large stresses also affect the modulus, but these changes are reversed by heating above $0.5 \mathrm{~K}$, suggesting that defects introduced by stress are easier to anneal than those produced during growth.
\end{abstract}

PACS: 67.80.bd Superfluidity in solid ${ }^{4} \mathrm{He}$, supersolid ${ }^{4} \mathrm{He}$; 67.80.dj Defects, impurities, and diffusion; 67.80.de Structure, lattice dynamics and sound.

Keywords: solid helium, supersolid, quantum solid, dislocations, shear modulus, annealing, plastic deformation.

\section{Introduction}

Helium is a uniquely quantum solid and recent torsional oscillator (TO) measurements [1-3] on hcp ${ }^{4} \mathrm{He}$ provide evidence for a dramatic new phenomenon at low temperatures - «supersolidity». Although several other groups [4-8] have now observed TO period shifts attributed to decoupling or «nonclassical rotational inertia» (NCRI), other phenomena like pressure-induced superflow $[9,10]$ or second sound modes [11] have not been seen. The NCRI fraction in TO measurements varies widely between different oscillators and depends on how crystals are grown and annealed [5,6], indicating that defects are involved. Theoretical work also suggests that supersolidity does not occur in a perfect crystal $[12,13]$ and that vacancies [14], grain boundaries [15], glassy regions [16] or dislocations [17,18] are involved.

Dislocations are produced during crystal growth (particularly constant volume or «blocked capillary» growth) or by plastic deformation, and can be partially eliminated by annealing near the melting temperature. They can dramatically affect elastic properties and may lead to anomalous low-temperature behavior in quantum crystals like helium $[19,20]$. We have recently made low-frequency measurements [21] of the shear modulus, $\mu$, of solid ${ }^{4} \mathrm{He}$ using a new technique in which piezoelectric transducers are directly embedded in the helium to generate shear strains and measure the resulting stresses. We observed large, unexpected increases in $\mu$ over the temperature range where decoupling was observed in TO experiments. Figure 1 shows the shear modulus (measured at $2000 \mathrm{~Hz}$ ) in two hcp ${ }^{4} \mathrm{He}$ crystals grown using the blocked capillary method (the 29.3 bar crystal passed along the bcc/hcp line in the ${ }^{4} \mathrm{He}$ phase diagram during growth). Below $200 \mathrm{mK}$, the shear modulus increases by $\Delta \mu \sim 11 \%(8 \%)$ in the 29.3 (33.3) bar crystal; similar stiffening was seen in all 8 samples we studied, with $\Delta \mu$ varying by a factor of about two. For comparison, the upper curve is torsional oscillator data (at $910 \mathrm{~Hz}$ ) for a typical 65 bar crystal [1], showing a $1.5 \%$ NCRI fraction with a very similar temperature dependence. The magnitudes of the modulus changes were independent of frequency (over our measurement range 20 to $2000 \mathrm{~Hz}$ ) but decreased at large strain amplitudes and shifted to lower temperatures at lower frequencies and when the ${ }^{3} \mathrm{He}$ concentration was reduced from $0.3 \mathrm{ppm}$ to $1 \mathrm{ppb}$. This behavior is nearly identical to that 


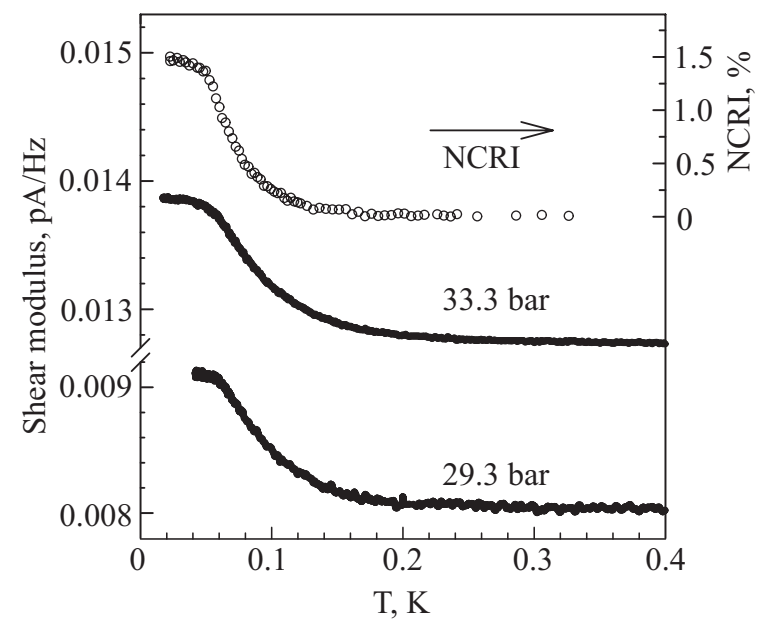

Fig. 1. Shear modulus of solid hcp ${ }^{4} \mathrm{He}$ at 29.3 and 33.3 bar showing anomalous increase below $200 \mathrm{mK}$. The upper curve (open symbols, right axis) shows the NCRI fraction from torsional oscillator measurements at 65 bar [1] for comparison.

of the NCRI measured in TO experiments and the two phenomena must be closely related.

We interpret our shear modulus anomaly in terms of dislocation motion. Dislocations form three-dimensional networks, pinned at nodes where they intersect, and are characterized by their Burgers vector $\mathbf{b}$ (an interatomic spacing), density $\Lambda$ (total dislocation length per unit volume) and network loop length between nodes $L_{N}$. When an external stress $\sigma$ is applied, dislocations experience a driving force $R b \sigma$, where the orientation factor $R$ (which relates the total stress to the shear component in the glide plane) varies between 0 and 0.5 . At low frequencies, inertia and damping are not important and dislocations simply bow out between pinning points, creating an additional strain and reducing the solid's shear modulus. In this regime the modulus change due to dislocations is independent of frequency [22]:

$$
\frac{\Delta \mu}{\mu}=-C R \Lambda L^{2},
$$

where $C$ is a constant which depends on the distribution of loop lengths $(\sim 0.1$ for a single length, $\sim 0.5$ for an exponential distribution with average length $L$ ). In the absence of impurity pinning, $L$ is the network length $L_{N}$, which is largest for low density dislocation networks with few intersections. In annealed crystals with well-defined networks, $\Lambda L_{N}^{2}$ is a geometric constant (e.g., 3 for a cubic network) and $\Delta \mu$ can be nearly independent of the dislocation density. For random orientations, the average anisotropy factor is about 0.2 , so dislocations can reduce $\mu$ by as much as $30 \%$.

Impurities are attracted to dislocations with a binding energy $E_{B}$ (for ${ }^{3} \mathrm{He}$ in hcp ${ }^{4} \mathrm{He}, E_{B} \sim 0.3$ to $0.7 \mathrm{~K}[23,24]$ ). They can pin dislocations very effectively at low tempera- tures (where the impurity pinning length $L_{P}$ is smaller than $L_{N}$ ), reducing the dislocation strain and increasing the shear modulus toward its intrinsic value. The increase in $\mu$, its magnitude and frequency dependence, the temperature at which stiffening occurs and its dependence on ${ }^{3} \mathrm{He}$ concentration, the amplitude dependence and its associated hysteresis - these are all consistent with a picture of a network of dislocations pinned at low temperatures by ${ }^{3} \mathrm{He}$ impurities (using dislocation parameters determined in earlier experiments [25-28] on hcp ${ }^{4} \mathrm{He}$ ).

The shear modulus anomaly can be explained by dislocation motion and is clearly connected to the NCRI seen in TO measurements, implying that such defects play an important role in possible supersolidity. It is important to understand how the behavior depends on dislocation density and other parameters. The TO measurements [6,7] which show the largest NCRI involve rapid blocked capillary growth in the narrow gap of an annulus, conditions which would be expected to produce high defect densities. Annealing of these crystals reduced the NCRI dramatically, presumably by eliminating defects. However, annealing had much smaller effects in other TO measurements, even increasing the NCRI in some cases. In our experiments we have tried to change the defect density in two ways. Annealing crystals near melting should eliminate some defects, while applying large stresses could add defects.

\section{Experimental results and discussion}

The standard blocked capillary technique involves growing crystals at constant volume, which produces large pressure changes as growth proceeds and leaves significant gradients after solidification is complete. These gradients can be reduced or eliminated by annealing near melting. Figure 2 shows the pressure (measured with an in situ capacitive gauge) for a typical sample during freezing (open symbols) and subsequent melting (solid symbols). During cooling (at $1 \mathrm{mK} / \mathrm{min}$ ), freezing is complete near $1.85 \mathrm{~K}$, but the transition is rounded, indicating that there are initial pressure differences in the solid of at least 200 mbar. We cooled to $1.80 \mathrm{~K}$ and then warmed more slowly (at $0.5 \mathrm{mK} / \mathrm{min}$ ), so that the sample remained within $50 \mathrm{mK}$ of melting for roughly $3.5 \mathrm{~h}$. The melting transition was much sharper, indicating that the remaining pressure differences within the cell were less than 5 mbar. Much larger pressure changes (decreases of up to 7 bar) been seen in other experiments [7,29] when crystals are annealed near melting. The pressure changes could reflect overall pressure changes in the sample (e.g., as low density disordered regions crystallize) or could reflect the equalization of initial pressure/density gradients in the cell (e.g., leading to a pressure increase if the pressure gauge is in a location where the helium freezes last, at the lowest pressure). We believe that the difference between 


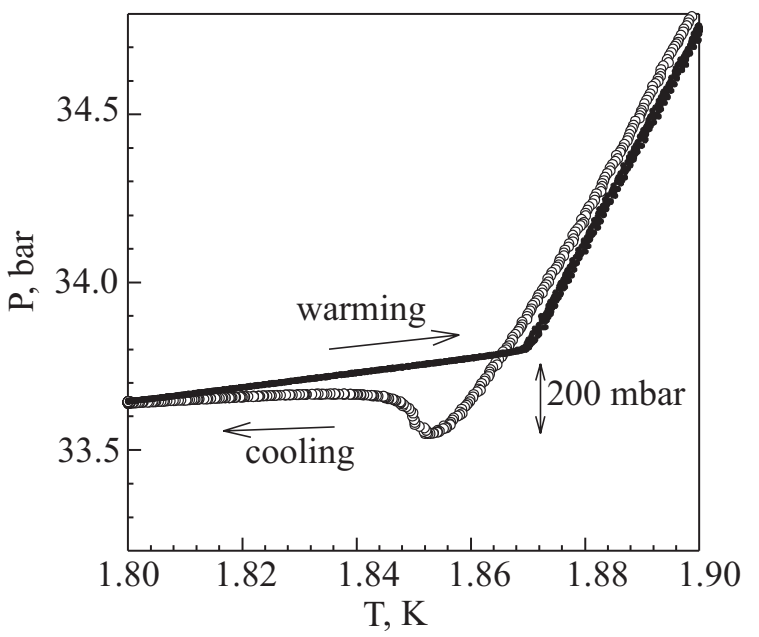

Fig. 2. Pressure measured in situ during blocked capillary freezing of ${ }^{4} \mathrm{He}$ (open symbols) and subsequent melting (solid symbols). Annealing essentially eliminates the initial pressure gradients ( $\sim 200$ mbar).

the cooling and melting curves in Fig. 2 are due to annealing of small pressure gradients in our rather open cell. Larger pressure changes would be expected in more confined geometries, e.g., in the narrow annulus of Ref. 7. The small offset between the cooling and warming pressures along the melting curve (above $1.87 \mathrm{~K}$ ) may simply reflect thermal lags in our system.

Plastic deformation also creates pressure gradients which can be reduced or eliminated by annealing near melting. We studied $[9,30]$ the flow of solid helium through the $25 \mu \mathrm{m}$ diameter channels of a glass capillary array by compressing the helium on one side and monitoring the resulting pressure changes on the other side. Figure 3 shows typical behavior for a 42 bar sample (melting temperature $T_{m}=2.12 \mathrm{~K}$ ) when a pressure difference of $\sim 130$ mbar was applied and then removed about $300 \mathrm{~s}$ later. At $2.10 \mathrm{~K}$ (solid symbols) the pressure stabilized within a minute following a compression and returned to its original value after decompression. At this temperature ( $20 \mathrm{mK}$ below melting), flow of solid helium eliminated pressure differences in the cell very quickly. The response depended strongly on temperature. The open symbols in Fig. 3 show the response at $2.00 \mathrm{~K}(120 \mathrm{mK}$ below melting). Although there is still flow, the increase is slower and the pressure stabilizes at a lower value. When the compression is released, the pressure decreases, but does not return to its original value. Annealing at this temperature does not completely eliminate pressure differences - only within about $50 \mathrm{mK}$ of melting is this possible.

Figure 4 shows the low amplitude shear modulus (measured [21] at $2000 \mathrm{~Hz}$ ) for isotopically pure (1 ppb ${ }^{3} \mathrm{He}$ ) solid ${ }^{4} \mathrm{He}$ at a pressure of 33.4 bar. During the initial cooling, the modulus increase at low temperature was about $13 \%$. After annealing at $1.70 \mathrm{~K}(150 \mathrm{mK}$ below the

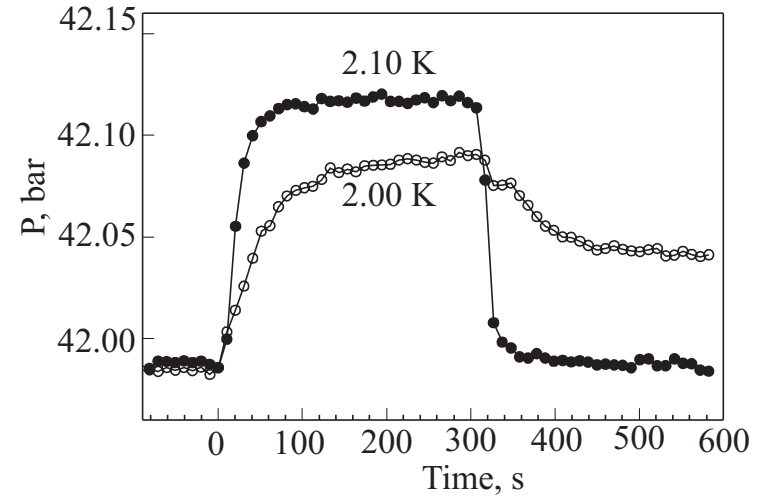

Fig. 3. Pressure response due to flow of hcp ${ }^{4} \mathrm{He}$ through glass capillaries following a compression and subsequent decompression. At $2.10 \mathrm{~K}(20 \mathrm{mK}$ below the melting temperature, $\left.T_{m}=2.12 \mathrm{~K}\right)$, the 130 mbar pressure difference equilibrates completely; at $2.00 \mathrm{~K}$, significant pressure differences ( $\sim 50 \mathrm{mbar}$ ) remain. Lines are guides to the eye.

melting temperature) for 11 hours, the increase was smaller, about $10 \%$. However, the low-temperature value of $\mu$ was essentially unchanged - it was the high-temperature modulus which increased by $3 \%$. This behavior supports our interpretation that the low-temperature value reflects the intrinsic (defect-free) modulus and the change in $\mu$ is due to pinned dislocations becoming mobile as ${ }^{3} \mathrm{He}$ impurities unbind at high temperatures. We made measurements on several commercial purity ${ }^{4} \mathrm{He}$ samples $\left(0.3 \mathrm{ppm}{ }^{3} \mathrm{He}\right)$ with similar results - annealing changed the high-temperature modulus by 1 to $3 \%$. The modulus usually increased but in one case annealing reduced it by $1 \%$. The annealing effects can be understood in terms of changes in dislocation density $\Lambda$; note that $\Delta \mu$ depends only on the combination $\Lambda \mathrm{L}^{2}$ which may increase,

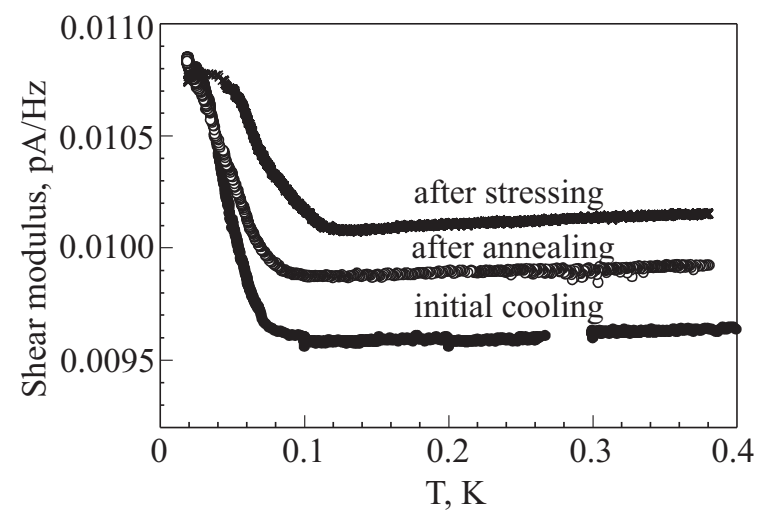

Fig. 4. Shear modulus in isotopically pure $\left(1 \mathrm{ppb}{ }^{3} \mathrm{He}\right) \mathrm{hcp}{ }^{4} \mathrm{He}$ at 33.4 bar. The lowest curve shows data from the initial cooling run. Annealing at $1.7 \mathrm{~K}$ for $11 \mathrm{~h}$ increased the modulus above $0.1 \mathrm{~K}$ but left the low-temperature value unchanged (middle curve). Applying a large acoustic stress at $19 \mathrm{mK}$ further increased the high-temperature modulus but again did not affect the value at low temperature (upper curve). 
decrease or remain the same when the dislocation density decreases. To eliminate the modulus anomaly entirely would require an almost dislocation free sample.

We also tried to introduce defects by applying large stresses to our samples at low temperature. Large ultrasonic pulses have been shown [31] to change the velocity and reduce the attenuation of longitudinal waves in solid helium, effects attributed to pinning of dislocations when they vibrate with large amplitudes. In our experiments, we generated stresses using acoustic resonances of the helium [21]. The resonance peaks were quite sharp at low temperature (quality factor $\mathrm{Q} \sim 2000$ at a resonant frequency $f_{r} \sim 8 \mathrm{kHz}$ ), allowing us to apply stresses much larger than the $\sim 0.3 \mathrm{~Pa}$ stress at which the modulus measurements were made. The upper curve in Fig. 4 is the modulus after an acoustic stress $\sim 700 \mathrm{~Pa}$ was applied at the lowest temperature $(19 \mathrm{mK})$. As after annealing, the low-temperature modulus was unaffected, but the high-temperature value changed, in this case increasing by $2 \%$ and further reducing the low-temperature modulus anomaly $\Delta \mu$. It also appears that the stresses broadened the transition, raising its onset temperature by about $30 \mathrm{mK}$. The response of other samples to large stresses was similar.

The changes induced by large acoustic stresses could be reversed by warming. The modulus of each stressed sample recovered to its original value around $0.5 \mathrm{~K}$. This is marked contrast to the annealing of pressure gradients produced by blocked capillary growth or by large plastic deformations - these could only be eliminated at temperatures much closer to melting. This suggests that different defects are introduced by the acoustic stresses. For example, the acoustic stresses could cause dislocations to intersect, producing jogs which might pin them, without creating the new dislocations that macroscopic plastic deformations would produce. By studying the kinetics of the recovery process, we hope to identify the mechanism responsible for the effects of large stresses.

\section{Summary}

Pressure and elastic measurements provide useful information about annealing of defects in solid helium. The pressure gradients produced during crystal growth or by plastic deformation can only be eliminated at temperatures very close to melting. However, our elastic shear modulus measurements show that even this does not produce defect-free crystals - dislocations still have a large effect on mechanical behavior of solid ${ }^{4} \mathrm{He}$. Annealing does affect the shear modulus stiffening which we observe below $200 \mathrm{mK}$, although the effect is not as dramatic as it sometimes is for decoupling in a torsional oscillator [6]. Large stresses also change the elastic behavior, perhaps by pinning dislocations. Our measurements suggest that eliminating the effects of dislocations on elastic or torsional oscillator experiments will require the growth of single crystals at constant pressure and care to avoid mechanical stresses afterward.

\section{Acknowledgement}

We would like to thank the Natural Sciences and Engineering Research Council of Canada and the University of Alberta for support of this research and M.H.W. Chan for providing the TO data of Fig. 1.

1. E. Kim and M.H.W. Chan, Science 305, 1941 (2004).

2. E. Kim and M.H.W. Chan, Phys. Rev. Lett. 97, 115302 (2006).

3. E. Kim and M.H.W. Chan, Nature 427, 225 (2004).

4. M. Kondo, S. Takada, Y. Shibayama, and K. Shirahama, J. Low Temp. Phys. 148, 695 (2007).

5. A. Penzev, Y. Yasuta, and M. Kubota, J. Low Temp. Phys. 148, 677 (2007).

6. A.S.C. Rittner and J.D. Reppy, Phys. Rev. Lett. 97, 165301 (2006).

7. A.S.C. Rittner and J.D. Reppy, Phys. Rev. Lett. 98, 175302 (2007).

8. Y. Aoki, J.C. Graves, and H. Kojima, Phys. Rev. Lett. 99, 015301 (2007).

9. J. Day and J. Beamish. Phys. Rev. Lett. 96, 105304 (2006).

10. J. Day, T. Herman, and J. Beamish, Phys. Rev. Lett. 95, 035301 (2005).

11. Y. Aoki, X. Lin, and H. Kojima, J. Low Temp. Phys. 148, 659 (2007).

12. D.M. Ceperley and B. Bernu, Phys. Rev. Lett. 93, 155303 (2004).

13. N. Prokof'ev and B. Svistunov, Phys. Rev. Lett. 94, 155302 (2005).

14. P.W. Anderson, W.F. Brinkman, and D.A. Huse, Science 310, 1164 (2005).

15. L. Pollet, M. Boninsegni, A.B. Kuklov, N.V. Prokof'ev, B.V. Svistunov, and M. Troyer, Phys. Rev. Lett. 98, 135301 (2007).

16. M. Boninsegni, N. Prokof'ev, and B. Svistunov, Phys. Rev. Lett. 96, 105301 (2006).

17. M. Boninsegni, A.B. Kuklov, L. Pollet, N.V. Prokof'ev, B.V. Svistunov, and M. Troyer, Phys. Rev. Lett. 99, 035301 (2007).

18. J. Toner, arXiv:0707.3842

19. P.-G. De Gennes, C. R. Physique 7, 561 (2006).

20. J.-P. Bouchard and G. Biroli, arxiv:0710.3087.

21. J. Day and J. Beamish, Nature 450, 853 (2007).

22. A. Granato and K. Lucke, J. Appl. Phys. 27, 583 (1956).

23. M.A. Paalanen, D.J. Bishop, and H.W Dail, Phys. Rev. Lett. 46, 664 (1981).

24. I. Iwasa and H. Suzuki, J. Phys. Soc. Jpn 49, 1722 (1980).

25. F. Tsuruoka and Y. Hiki, Phys. Rev. B20, 2702 (1979).

26. V.L. Tsymbalenko, Sov. Phys. JETP 47, 787 (1978).

27. I. Iwasa, K. Araki, and H. Suzuki, J. Phys. Soc. Jpn 46, 1119 (1979).

28. F. Tsuruoka and Y. Hiki, Phys. Rev. B20, 2702 (1979).

29. V. Grigor'ev, V.A. Maidanov, V.Yu. Rubanskii, S.P. Rubets, E.Ya. Rudavskii, A.S. Rybalko, Ye.Y. Syrnikov, and V.A. Tikhii, condmat 0702133 (2007).

30. J. Day and J. Beamish, J. Low Temp. Phys. 148, 683 (2007).

31. J.R. Beamish and J.P. Franck, Phys. Rev. B28, 1419 (1983). 